\title{
Maximum principle and existence of solutions for non-necessarily cooperative systems involving Schrödinger operators
}

\author{
H. M. Serag and A. H. Qamlo
}




\title{
MAXIMUM PRINCIPLE AND EXISTENCE OF SOLUTIONS FOR NON-NECESSARILY COOPERATIVE SYSTEMS INVOLVING SCHRÖDINGER OPERATORS
}

\author{
H. M. SERAG AND A. H. QAMLO
}

Received 13 February, 2006

\begin{abstract}
In this paper, we obtain the necessary and sufficient conditions for having the maximum principle and existence of positive solutions for some cooperative systems involving Schrödinger operators defined on unbounded domains. Then, we deduce the existence of solutions for semi-linear systems. Finally we discuss the generalized maximum principle ( $\phi_{q}$-positivity) for non-cooperative systems.
\end{abstract}

2000 Mathematics Subject Classification: 35P30, 35J70

Keywords: maximum principle, generalized maximum principle, positive solution, cooperative and non-cooperative semilinear systems, Schrödinger operators

\section{INTRODUCTION}

We consider the following semilinear system

$$
\left\{\begin{array}{l}
L Y+Q Y=A g(x) Y+F(x, Y) \text { in } \Omega, \\
Y \rightarrow 0 \text { as }|x| \rightarrow \infty \\
Y=0 \text { on } \partial \Omega
\end{array}\right.
$$

where $\Omega$ is an unbounded domain of $R^{N}, L$ is an $n \times n$ diagonal matrix of Laplace operators, $Q$ is an $n \times n$ diagonal matrix of potential functions $q_{i}(1 \leq i \leq n), g(x)$ is a weight function tending to zero at infinity, $F$ is a given $n$-vector function and $A=\left(a_{i j}\right)$ is a constant $n \times n$ cooperative matrix such that

$$
a_{i j} \geq 0 \text { for all } i \neq j .
$$

It is well known that the maximum principle plays an important role in the theory of partial differential equations. An analogous theory has been appeared for semilinear systems in [1,5-9,11,14,22]. In [7,9], the authors studied system (S) with $q_{i}=0$ and $g(x)=1$, defined on bounded domains with Dirichlet conditions. The problem with $q_{i}=0$ defined on the whole space $R^{N}$ has been established in $[12,13]$. The system with equal potentials defined on $R^{N}$ has been considered in $[3,4]$. 
Some applications concerning the optimal control of systems like (S) have been introduced in [15-21].

Here, we extend these results to system (S). In section two, we obtain necessary or sufficient conditions for having the maximum principle and existence of positive solutions for cooperative linear systems. Then, we study semilinear systems in section three; we adapt the method of sub and super solutions for proving the existence of nonnegative solutions. Finally, in section four, we study the generalized maximum principle ( $\varphi_{q}$-positivity) for non-cooperative systems.

To prove our theorems, we make use of earlier results by Djellit and Yechoui [10] who proved that, for $N>2$ and $q>0$, if there exist $\alpha>0, \beta \geq 1, \alpha>\beta$, and $k, c>0$ such that

$$
0<g(x) \leq \frac{k}{\left(1+|x|^{2}\right)^{\alpha}}, \quad 0<q(x) \leq \frac{c}{\left(1+|x|^{2}\right)^{\beta}},
$$

then the eigenvalue problem

$$
\left\{\begin{array}{l}
(-\Delta+q) y=\lambda g(x) y \quad \text { in } \quad \Omega \\
y \rightarrow 0 \text { as } \quad|x| \rightarrow \infty \\
y=0 \text { on } \partial \Omega
\end{array}\right.
$$

has simple principal eigenvalue $\left(\lambda_{q}^{+}\right)$which is associated with positive eigenfunction $\varphi_{q}$ on $V$.

Moreover $\left(\lambda_{q}^{+}\right)$is characterized by

$$
\lambda_{q}^{+} \int_{\Omega} g(x)|y|^{2} d x \leq|\nabla y|^{2}+q|y|^{2},
$$

where

$$
V(\Omega)=\left\{y \in D^{\prime}(\Omega)||\left(1+|x|^{2}\right)^{-\frac{1}{2}} y \in L^{2}(\Omega), \nabla y \in L^{2}(\Omega)\right\}
$$

is a Hilbert space with an inner product $(y, \psi)_{V}=\int_{\Omega}\left(\nabla y . \nabla \psi+\frac{1}{1+|x|^{2}} y \psi\right) d x$ and a norm

$$
\|y\|_{V}=\left(\int_{\Omega}\left(|\nabla y|^{2}+\frac{1}{1+|x|^{2}}|y|^{2}\right) d x\right)^{\frac{1}{2}}
$$

which is equivalent to

$$
\|y\|_{q}=\left(\int_{\Omega}\left(|\nabla y|^{2}+q|y|^{2}\right) d x\right)^{\frac{1}{2}} .
$$

We also introduce the Hilbert space

$$
\mathscr{H}=\left\{y: \Omega \rightarrow R \mid \int_{\Omega} g y^{2} d x \leq \infty\right\}=L_{g}^{2}(\Omega)
$$


with an inner product

$$
(y, \psi)_{g}=\int_{\Omega} g y \psi d x .
$$

\section{COOPERATIVE LINEAR SYSTEMS}

In this section, we study the maximum principle and existence of positive solutions for system $(S)$ when the right-hand side is linear.

Definition 1. We say that the maximum principle holds for system (S) if $F \geq 0$ and $Y$ is a solution of (S), then $Y \geq 0$.

Definition 2. A non-singular square matrix $B=\left(b_{i j}\right)$ is said to be an $M$-matrix if $b_{i j} \leq 0$ for $i \neq j, b_{i i}>0$ for $i=1, \ldots, n$, and if all the principal minors extracted from $B$ are positive.

The $i$ th equation of system (S) can be written as

$$
\left\{\begin{array}{l}
\left(-\Delta+q_{i}\right) y_{i}=g(x) \sum_{j=1}^{n} a_{i j} y_{j}+f_{i} \quad \text { in } \Omega, \\
y_{i} \rightarrow 0 \quad \text { as } \quad|x| \rightarrow \infty \\
y_{i}=0 \quad \text { on } \quad \partial \Omega .
\end{array}\right.
$$

Theorem 1. Assume that (1) and (2) with $q=q_{i}$ hold, and $f_{i} \geq 0$. System (S) satisfies the maximum principle if

the matrix $\left(\Lambda_{Q}^{+}-A\right)$ is a non-singular $M$-matrix, where

$$
\Lambda_{Q}^{+}=\left(\begin{array}{cccc}
\lambda_{q_{1}}^{+} & 0 & \ldots & 0 \\
0 & \lambda_{q_{2}}^{+} & \cdots & 0 \\
\vdots & \vdots & \ddots & \vdots \\
0 & 0 & \ldots & \lambda_{q_{n}}^{+}
\end{array}\right)
$$

Moreover, if the maximum principle holds for system (S), then

the matrix $\left(\Lambda_{q}^{+}-A\right)$ is a non-singular $M$-matrix, where

$$
q=\max \left\{q_{i}: 1 \leq i \leq n\right\}, \quad \Lambda_{q}^{+}=\left(\begin{array}{cccc}
\lambda_{q}^{+} & 0 & \ldots & 0 \\
0 & \lambda_{q}^{+} & \ldots & 0 \\
\vdots & \vdots & \ddots & \vdots \\
0 & 0 & \ldots & \lambda_{q}^{+}
\end{array}\right) \text {. }
$$

Proof. First assume that $f_{i} \geq 0$ and $\left(y_{i}\right)_{i=1}^{n} \in \prod_{i=1}^{n} V_{q_{i}}$ is a solution of (S). Multiplying $\left(S_{i}\right)$ by $y_{i}^{-}=\max \left\{-y_{i}, 0\right\}$ and integrating over $\Omega$, we obtain by Green's 
formula that

$$
\int_{\Omega} \nabla y_{i} \cdot \nabla y_{i}^{-} d x+\int_{\Omega} q_{i} y_{i} y_{i}^{-} d x=\sum_{j=1}^{n} a_{i j} \int_{\Omega} g(x) y_{j} y_{i}^{-} d x+\int_{\Omega} f_{i} y_{i}^{-} d x,
$$

i.e.,

$$
\begin{aligned}
\int_{\Omega}\left|\nabla y_{i}^{-}\right|^{2} d x & +\int_{\Omega} q_{i}\left|y_{i}^{-}\right|^{2} d x \\
& =a_{i i} \int_{\Omega} g(x)\left|y_{i}^{-}\right|^{2} d x-\sum_{j \neq i}^{n} a_{i j} \int_{\Omega} g(x) y_{j} y_{i}^{-} d x-\int_{\Omega} f_{i} y_{i}^{-} d x,
\end{aligned}
$$

and thus, by (3), we get

$$
\begin{aligned}
\lambda_{q_{i}}^{+} \int_{\Omega} g(x)\left|y_{i}^{-}\right|^{2} d x & \\
& \leq a_{i i} \int_{\Omega} g(x)\left|y_{i}^{-}\right|^{2} d x-\sum_{j \neq i}^{n} a_{i j} \int_{\Omega} g(x) y_{j} y_{i}^{-} d x-\int_{\Omega} f_{i} y_{i}^{-} d x .
\end{aligned}
$$

Therefore

$$
\left(\lambda_{q_{i}}^{+}-a_{i i}\right) \int_{\Omega}\left|\sqrt{g} y_{i}^{-}\right|^{2} d x \leq \sum_{j \neq i}^{n} a_{i j} \int_{\Omega} g(x) y_{j}^{-} y_{i}^{-} d x
$$

and, by the Cauchy-Schwartz inequality, we have

$$
\left(\lambda_{q_{i}}^{+}-a_{i i}\right)\left(\int_{\Omega}\left|\sqrt{g} y_{i}^{-}\right|^{2} d x\right)^{\frac{1}{2}}-\sum_{j \neq i}^{n} a_{i j}\left(\int_{\Omega}\left|\sqrt{g} y_{j}^{-}\right|^{2} d x\right)^{\frac{1}{2}} \leq 0,
$$

which can be rewritten to the form

$$
\left(\begin{array}{cccc}
\lambda_{q_{1}}^{+}-a_{11} & -a_{12} & \cdots & -a_{1 n} \\
-a_{21} & \lambda_{q_{2}}^{+}-a_{22} & \cdots & -a_{2 n} \\
\vdots & \vdots & \ddots & \vdots \\
-a_{n 1} & -a_{n 2} & \cdots & \lambda_{q_{n}}^{+}-a_{n n}
\end{array}\right)\left(\begin{array}{c}
\left(\int_{\Omega}\left|\sqrt{g} y_{1}^{-}\right|^{2} d x\right)^{1 / 2} \\
\left(\int_{\Omega}\left|\sqrt{g} y_{2}^{-}\right|^{2} d x\right)^{1 / 2} \\
\vdots \\
\left(\int_{\Omega}\left|\sqrt{g} y_{n}^{-}\right|^{2} d x\right)^{1 / 2}
\end{array}\right) \leq 0
$$

Now, (4), $y_{1}^{-}=y_{2}^{-}=\cdots=y_{n}^{-}=0$ and hence $y_{1}, y_{2}, \ldots, y_{n} \geq 0$. 
Assume now that $0 \leq f_{i} \in L_{1 / g}^{2}(\Omega)$ and that the maximum principle holds for system $(S)$. We rewrite $\left(S_{i}\right)$ as follows:

$$
\left\{\begin{array}{l}
(-\Delta+q) y_{i}=g(x) \sum_{j=1}^{n} a_{i j} y_{j}+H_{i} \quad \text { in } \quad \Omega \\
y_{i} \rightarrow 0 \text { as } \quad|x| \rightarrow \infty \\
y_{i}=0 \quad \text { on } \quad \partial \Omega
\end{array}\right.
$$

where $0 \leq H_{i}=\left(q-q_{i}\right) y_{i}+f_{i} \in L_{1 / g}^{2}(\Omega)$.

Multiplying by $\varphi_{q}$ (the eigenfunction corresponding $\lambda_{q}^{+}$) and integrating over $\Omega$, we get

$$
\int_{\Omega}(-\Delta+q) y_{i} \varphi_{q} d x=\sum_{j=1}^{n} a_{i j} \int_{\Omega} g(x) y_{j} \varphi_{q} d x+\int_{\Omega} H_{i} \varphi_{q} d x
$$

and, by using Green's formula and (3), we obtain

$$
\left(\lambda_{q}^{+}-a_{i i}\right) \int_{\Omega} g(x) y_{i} \varphi_{q} d x-\sum_{j \neq i}^{n} a_{i j} \int_{\Omega} g(x) y_{j} \varphi_{q} d x=\int_{\Omega} H_{i} \varphi_{q} d x
$$

which is a Cramer system in $X_{i}=\int_{\Omega} g(x) y_{i} \varphi_{q} d x, 1 \leq i \leq n$. Since the right-hand side is non-negative as well as $X_{i}$, we obtain

$$
\left|\begin{array}{cccc}
\lambda_{q}^{+}-a_{11} & -a_{12} & \cdots & -a_{1 n} \\
-a_{21} & \lambda_{q}^{+}-a_{22} & \cdots & -a_{2 n} \\
\vdots & \vdots & \ddots & \vdots \\
-a_{n 1} & -a_{n 2} & \cdots & \lambda_{q}^{+}-a_{n n}
\end{array}\right|=\left|\Lambda_{q}^{+}-A\right|>0 .
$$

The inequality $\lambda_{q}^{+}-a_{i i}>0$ is satisfied from the scalar case (see [13]), because the functions $g, y_{i}, H_{i}$ and the coefficients $a_{i j}$ for $i \neq j$ are non-negative.

Remark 1. If $q_{i}=q$ for $1 \leq i \leq n$, then condition (6) is the necessary and sufficient condition for having the maximum principle for system (S).

Theorem 2. Let (1) and (2) with $q=q_{i}$ hold. Then, for $F \geq 0$, system (S) has a unique positive solution if condition (4) is satisfied.

Proof. We consider the bilinear form $a: \prod_{i=1}^{n} V_{q_{i}} \times \prod_{i=1}^{n} V_{q_{i}} \rightarrow R$ defined by

$$
\begin{aligned}
a(Y, \Psi) & =a\left(\left(y_{1}, y_{2}, \ldots, y_{n}\right),\left(\psi_{1}, \psi_{2}, \ldots, \psi_{n}\right)\right) \\
& =\sum_{i=1}^{n} \int_{\Omega} \nabla y_{i} . \nabla \psi_{i} d x+\sum_{i=1}^{n} \int_{\Omega} q_{i} y_{i} \psi_{i} d x
\end{aligned}
$$




$$
-\sum_{j \neq i}^{n} \int_{\Omega} g\left(x a_{i j} y_{j} \psi_{i} d x-\sum_{i=1}^{n} \int_{\Omega} g(x) a_{i i} y_{i} \psi_{i} d x\right.
$$

We choose $m \geq 0$ such that $m+a_{i i}>0$. Then, we have

$$
\begin{aligned}
a(Y, Y)= & \sum_{i=1}^{n} \int_{\Omega}\left[\left|\nabla y_{i}\right|^{2}+q_{i} y_{i}^{2}\right] d x \\
& -\sum_{j \neq i}^{n} \int_{\Omega} g(x) a_{i j} y_{i} y_{j} d x-\sum_{i=1}^{n} \int_{\Omega} g(x) a_{i i} y_{i}^{2} d x \\
= & \sum_{i=1}^{n} \int_{\Omega}\left[\left|\nabla y_{i}\right|^{2}+\left(q_{i}+m g\right) y_{i}^{2}\right] d x \\
& -\sum_{j \neq i}^{n} \int_{\Omega} g(x) a_{i j} y_{i} y_{j} d x-\sum_{i=1}^{n}\left(m+a_{i i}\right) \int_{\Omega} g(x) y_{i}^{2} d x
\end{aligned}
$$

and, by the Cauchy-Schwartz inequality and (3), we get

$$
\begin{aligned}
a(Y, Y) \geq & \sum_{i=1}^{n} \int_{\Omega}\left[\left|\nabla y_{i}\right|^{2}+\left(q_{i}+m g\right) y_{i}^{2}\right] d x \\
& -\sum_{j \neq i}^{n} a_{i j}\left(\int_{\Omega}\left(\sqrt{g} y_{i}\right)^{2} d x\right)^{\frac{1}{2}}\left(\int_{\Omega}\left(\sqrt{g} y_{j}\right)^{2} d x\right)^{\frac{1}{2}} \\
& -\sum_{i=1}^{n}\left(m+a_{i i}\right) \int_{\Omega} g(x) y_{i}^{2} d x \\
\geq & \sum_{i=1}^{n}\left(1-\frac{m+a_{i i}}{m+\lambda_{q_{i}}^{+}}\right) \int_{\Omega}\left[\left|\nabla y_{i}\right|^{2}+\left(q_{i}+m g\right) y_{i}^{2}\right] d x \\
& -\sum_{j \neq i}^{n} \frac{a_{i j}}{\sqrt{\left(m+\lambda_{q_{i}}^{+}\right)\left(m+\lambda_{q_{j}}^{+}\right)}}\left(\int_{\Omega}\left[\left|\nabla y_{i}\right|^{2}+\left(q_{i}+m g\right) y_{i}^{2}\right] d x\right)^{\frac{1}{2}} \\
& \times\left(\int_{\Omega}\left[\left|\nabla y_{j}\right|^{2}+\left(q_{j}+m g\right) y_{j}^{2}\right] d x\right)^{\frac{1}{2}} .
\end{aligned}
$$

Therefore, using (4), it follows that

$$
a(Y, Y) \geq C \sum_{i=1}^{n} \int_{\Omega}\left[\left|\nabla y_{i}\right|^{2}+\left(q_{i}+m g\right)\left(y_{i}\right)^{2}\right] d x
$$




$$
\geq C \sum_{i=1}^{n} \int_{\Omega}\left[\left|\nabla y_{i}\right|^{2}+q_{i}\left(y_{i}\right)^{2}\right] d x=C \sum_{i=1}^{n}\left\|y_{i}\right\|_{q_{i}}^{2},
$$

where $C>0$. Then, by the Lax Milgram lemma, since $a$ is a continuous coercive bilinear form, there exists a unique solution $Y=\left(y_{i}\right)_{i=1}^{n} \in \prod_{i=1}^{n} V_{q_{i}}$. This solution is non-negative by the maximum principle.

\section{SEMILINEAR SYSTEMS}

In this section, we adapt the method of sub and super solutions, to prove the existence of solutions for semilinear cooperative system (S). The proof here is analogous to that of $[3,13]$.

We assume that $f_{i}(x, Y)=f_{i}\left(x, y_{1}, y_{2}, \ldots, y_{n}\right)$ is a Carathéodory function such that

$$
0 \leq F(x, Y)=\left(f_{1}(x, Y), f_{2}(x, Y), \ldots, f_{n}(x, Y)\right) \leq N g(x) Y+h^{\prime}
$$

for all $Y \geq 0$ and $x \in \Omega$, where $N$ is a positive constant and $0<h^{\prime}=(h, h, \ldots, h)$ is a bounded vector-function in $\left(L_{1 / g}^{2}(\Omega)\right)^{n}$.

Theorem 3. Let (1), (2) with $q=q_{i}$, and (8) be satisfied. Then there exists a positive solution of system $(\mathrm{S})$ if

the matrix $\left(\Lambda_{Q}^{+}-(A+N I)\right)$ is a non-singular $M$-matrix,

where $\Lambda_{Q}^{+}$is defined by the relation (5) and I denotes the identity matrix.

Proof. We divide the proof into several steps.

Step (i): Construction of sub and super solutions. It is clear that

$$
Y^{0}=\left(y_{1}^{0}, y_{2}^{0}, \ldots, y_{n}^{0}\right)=(0,0, \ldots, 0)
$$

is a sub solution of $(\mathrm{S})$, because

$$
L Y^{0}+Q Y^{0}-g(x) A Y^{0}-F\left(x, Y^{0}\right) \leq 0 .
$$

Consider now the system

$$
\left\{\begin{array}{l}
L Y+Q Y=g(x)(A+N I) Y+h^{\prime} \text { in } \Omega . \\
Y \rightarrow 0 \text { as }|x| \rightarrow \infty, \\
Y=0 \text { on } \partial \Omega,
\end{array}\right.
$$

It follows from Theorem 2 that, under condition (9), system (10) has a unique positive solution

By (8), we have

$$
Y^{*}=\left(y_{1}^{*}, y_{2}^{*}, \ldots, y_{n}^{*}\right) \text {. }
$$

$$
0 \leq(L+Q) Y^{*}-g(x) A Y^{*}-F\left(x, Y^{*}\right),
$$

i. e., $Y^{*}=\left(y_{1}^{*}, y_{2}^{*}, \ldots, y_{n}^{*}\right)$ is a super solution of $(\mathrm{S})$. 
Step (ii): Definition of a compact operator. We introduce

$$
T: \mathscr{H}^{n} \ni Y=\left(\begin{array}{c}
y_{1} \\
\vdots \\
y_{n}
\end{array}\right) \geq 0 \mapsto \Psi=\left(\begin{array}{c}
\psi_{1} \\
\vdots \\
\psi_{n}
\end{array}\right)=T Y \in \prod_{i=1}^{n} V_{q_{i}},
$$

where $\Psi$ is a unique solution of the following system:

$$
\left\{\begin{array}{l}
(L+Q+m g(x) I) \Psi=(m I+A) g(x) Y+F(x, Y) \text { in } \Omega, \\
\Psi \rightarrow 0 \text { as }|x| \rightarrow \infty, \\
\Psi=0 \text { on } \partial \Omega,
\end{array}\right.
$$

where $m \geq 0$ is such that $m+a_{i i}>0$. Since the equation in (12) can be rewritten as

$$
(L+Q) \Psi=-m g(x) \Psi+\bar{F}, \quad \bar{F}=(m I+A) g(x) Y+F(x, Y) \geq 0,
$$

system (12) has a unique solution $\Psi \in \prod_{i=1}^{n} V_{q_{i}}$ and therefore $T$ is well-defined.

Step (iii): $K=\left[Y^{0}, Y^{*}\right]$ is invariant by $T$, i.e., $T(K) \subseteq K$. For every $Y \in$ $\prod_{i=1}^{n} V_{q_{i}}, Y \geq 0$, we have $T(Y)=\Psi \geq 0$. We show now that if $Y \leq Y^{*}$, then $\Psi \leq Y^{*}$. From (10) and (12), we obtain

$$
(L+Q+m g(x) I)\left(Y^{*}-\Psi\right)=\tau,
$$

where $\tau:=(m I+A) g(x)\left(Y^{*}-Y\right)+N g(x) Y^{*}-F(x, Y) \geq 0$. Therefore

$$
(L+Q)\left(Y^{*}-\Psi\right)=-m g(x)\left(Y^{*}-\Psi\right)+\tau
$$

and thus $Y^{*}-\Psi$ is non-negative, i. e., $\Psi \leq Y^{*}$.

Step (iv): $T$ is a continuous operator. Let $Y_{k} \rightarrow Y$ in $\mathscr{H}^{n}$. Then we get

$$
F\left(x, Y_{k}\right) \rightarrow F(x, Y) \text { in }\left(\mathscr{H}^{\prime}\right)^{n} .
$$

If we denote $\Psi_{k}=T\left(Y_{k}\right)$, by (12) it follows that

$$
(L+Q+m g(x) I)\left(\Psi-\Psi_{k}\right)=(m I+A) g(x)\left(Y-Y_{k}\right)+F(x, Y)-F\left(x, Y_{k}\right) .
$$

Multiplying by $\left(\Psi-\Psi_{k}\right)$ and integrating over $\Omega$, we get

$$
\begin{aligned}
& \int_{\Omega}(L+Q+m g(x) I)\left(\Psi-\Psi_{k}\right) \cdot\left(\Psi-\Psi_{k}\right) d x \\
& =(m I+A) \int_{\Omega} g(x)\left(Y-Y_{k}\right) \cdot\left(\Psi-\Psi_{k}\right) d x \\
& \quad+\int_{\Omega}\left(F(x, Y)-F\left(x, Y_{k}\right)\right) \cdot\left(\Psi-\Psi_{k}\right) d x,
\end{aligned}
$$

which, by virtue of the Green formula, yields that

$$
\int_{\Omega}\left|\nabla\left(\Psi-\Psi_{k}\right)\right|^{2} d x+\int_{\Omega} Q\left|\Psi-\Psi_{k}\right|^{2} d x+m \int_{\Omega} g(x)\left|\Psi-\Psi_{k}\right|^{2} d x
$$




$$
\begin{aligned}
=(m I+A) \int_{\Omega} g(x)\left(Y-Y_{k}\right) \cdot\left(\Psi-\Psi_{k}\right) d x \\
\quad+\int_{\Omega}\left(F(x, Y)-F\left(x, Y_{k}\right) \mid\right) \cdot\left(\Psi-\Psi_{k}\right) d x .
\end{aligned}
$$

Now, using the Cauchy-Schwarz inequality, we get

$$
\begin{aligned}
\int_{\Omega}\left|\nabla\left(\Psi-\Psi_{k}\right)\right|^{2} d x & +\int_{\Omega} Q\left|\Psi-\Psi_{k}\right|^{2} d x+m \int_{\Omega} g(x)\left|\Psi-\Psi_{k}\right|^{2} d x \\
\leq(m I+A) \| Y & -Y_{k}\left\|_{\mathscr{H}^{n}}\right\| \Psi-\Psi_{k} \|_{\mathscr{H}^{n}} \\
& +\left\|F(x, Y)-F\left(x, Y_{k}\right)\right\|_{\left(\mathscr{H}^{\prime}\right)^{n}}\left\|\Psi-\Psi_{k}\right\|_{\mathscr{H}^{n}}
\end{aligned}
$$

and thus

$$
\begin{aligned}
\left\|\Psi-\Psi_{k}\right\|_{Q}^{2} \leq(m I+A)\left\|Y-Y_{k}\right\|_{\mathscr{H}^{n}}\left\|\Psi-\Psi_{k}\right\|_{\mathscr{H}^{n}} & \\
& +\left\|F(x, Y)-F\left(x, Y_{k}\right)\right\|_{\left(\mathscr{H}^{\prime}\right)^{n}}\left\|\Psi-\Psi_{k}\right\|_{\mathscr{H}^{n}},
\end{aligned}
$$

where $\|\cdot\|_{Q}$ denotes the norm in $\prod_{i=1}^{n} V_{q_{i}}$. Since $\left\|Y-Y_{k}\right\|_{\mathscr{H}} \rightarrow 0$, we have $\| \Psi-$ $\Psi_{k} \|_{Q} \rightarrow 0$.

Step (v): $T$ is a compact operator. First note the following: Let $Y \geq 0$ and $\Psi=$ $T(Y)$. Multiplying (12) by $\Psi$ and integrating over $\Omega$, we get

$$
\begin{aligned}
\int_{\Omega}|\nabla \Psi|^{2} d x+\int_{\Omega} Q|\Psi|^{2} d x & +m \int_{\Omega} g(x)|\Psi|^{2} d x \\
& =(m I+A) \int_{\Omega} g(x) Y \Psi d x+\int_{\Omega} F(x, Y) \Psi d x,
\end{aligned}
$$

which, in view of (8), yields that

$$
\begin{aligned}
& \int_{\Omega}|\nabla \Psi|^{2} d x+\int_{\Omega} Q|\Psi|^{2} d x+m \int_{\Omega} g(x)|\Psi|^{2} d x \\
& \leq(m I+A) \int_{\Omega} g(x) Y \Psi d x+N \int_{\Omega} g(x) Y \Psi d x+\int_{\Omega} \sqrt{g} \Psi \frac{h}{\sqrt{g}} d x \\
& =(m I+A+N I) \int_{\Omega} g(x) Y \Psi d x+\int_{\Omega} \sqrt{g} \Psi \frac{h}{\sqrt{g}} d x .
\end{aligned}
$$

By the Cauchy-Schwarz inequality, we obtain

$$
\|\psi\|_{Q} \leq C\left(\|Y\|_{(\mathscr{H})^{n}}+\|h\|_{\mathscr{H}^{\prime}}\right) \leq C\left(\|Y\|_{Q}+\|h\|_{\mathscr{H}^{\prime}}\right)
$$

Therefore if $\left\{Y_{k}\right\}_{k \in N}$ is a bounded sequence in $\prod_{i=1}^{n} V_{q_{i}}$, the associated sequence $\left\{\Psi_{k}\right\}_{k \in N}$ is bounded in $\prod_{i=1}^{n} V_{q_{i}}$. We show now that $\left\{\Psi_{k}\right\}_{k \in N}$ is a Cauchy sequence in $\prod_{i=1}^{n} V_{q_{i}}$. 
Suppose that $\left\|Y_{k}\right\|_{Q}^{2} \leq E$ for $k \in N, E$ is a constant. In view of (2), we can choose $R$ large enough such that

$$
\left(1+|x|^{2}\right) g(x)<\frac{\epsilon}{8 \gamma E} \quad \text { for } \quad|x| \geq R,
$$

where $\epsilon>0$ is fixed and $\gamma$ is given by

$$
\int_{\Omega}\left(1+|x|^{2}\right)^{-1} y^{2} d x \leq \gamma \int_{\Omega}|\nabla y|^{2} d x .
$$

Let $B=\{x \in \Omega|| x \mid<R\}$ and $B^{\prime}=\{x \in \Omega|| x \mid \geq R\}$. Since $\left\{Y_{k}\right\}_{k \in N}$ is bounded in $\prod_{i=1}^{n} V_{q_{i}}, Y_{k}$ is bounded in $(\mathscr{H}(B))^{n}$. However $B$ is bounded and therefore the embedding $(\mathscr{H}(B))^{n}$ into $\left(L^{2}(B)\right)^{n}$ is compact. Hence there exists a convergent subsequence still denoted by $\left\{Y_{k}\right\}_{k \in N}$, which is a Cauchy sequence and thus, for every $j$ and $k$ large enough, we have

$$
\int_{B} g(x)\left|Y_{k}-Y_{j}\right|^{2} d x \leq \int_{B}\left|Y_{k}-Y_{j}\right|^{2} d x<\frac{\epsilon}{2} .
$$

Moreover using (13) and (14), we obtain

$$
\begin{aligned}
\int_{B^{\prime}} g(x)\left|Y_{k}-Y_{j}\right|^{2} d x & =\int_{B^{\prime}}\left(1+|x|^{2}\right) g(x) \frac{1}{1+|x|^{2}}\left|Y_{k}-Y_{j}\right|^{2} d x \\
& \leq \frac{\varepsilon}{8 \gamma E} \int_{B^{\prime}} \frac{1}{1+|x|^{2}}\left|Y_{k}-Y_{j}\right|^{2} d x \\
& \leq \frac{\varepsilon}{8 \gamma E} \gamma\left\|Y_{k}-Y_{j}\right\|_{Q}^{2}
\end{aligned}
$$

so $\int_{B^{\prime}} g(x)\left|Y_{k}-Y_{j}\right|^{2} d x<\frac{\epsilon}{2}$.

Then $\left\{\Psi_{k}\right\}_{k \in N}$ is a Cauchy sequence in $\prod_{i=1}^{n} V_{q_{i}}$. Hence it converges to $\Psi$ and therefore $T$ is compact in $\prod_{i=1}^{n} V_{q_{i}}$. By Schauder fixed point theorem, there exists at least one positive solution $Y=\left(y_{i}\right)_{i=1}^{n} \in \prod_{i=1}^{n} V_{q_{i}}$ of system (S) satisfying $Y^{0} \leq$ $Y \leq Y^{*}$.

\section{NON-COOPERATIVE SYSTEMS}

In this section, we study the generalized maximum principle ( $\varphi_{q}$-positivity) for $n \times n$ non-cooperative systems.

Definition 3. System (S) (with $q_{i}=q$ ) satisfies the generalized maximum principle if $F \geq 0$ and $Y=\left(y_{1}, y_{2}, \ldots, y_{n}\right)$ is a solution of (S), then there exists $C>0$ such that

$$
y_{i} \geq C \varphi_{q} \quad \text { in } \quad \Omega \quad \text { for all } \quad i=1,2, \ldots, n
$$


We start with $2 \times 2$ non cooperative systems:

$$
\left\{\begin{array}{l}
L_{q} y_{1}=a_{11} g(x) y_{1}+a_{12} g(x) y_{2}+f_{1} \text { in } \Omega, \\
L_{q} y_{2}=a_{21} g(x) y_{1}+a_{22} g(x) y_{2}+f_{2} \text { in } \Omega, \\
y_{1}, y_{2} \rightarrow 0 \text { as }|x| \rightarrow \infty, \\
y_{1}=y_{2}=0 \text { on } \partial \Omega,
\end{array}\right.
$$

As in [2], we can prove

Theorem 4. Assume that $a_{12}<0, a_{21}>0, a_{11}>a_{22}, f_{1}-\xi_{2} f_{2} \geq 0$, and ( $a_{11}-$ $\left.a_{22}\right)^{2}+4 a_{12} a_{21} \geq 0$ are satisfied. The generalized maximum principle holds for system $\left(S_{2}\right)$ if

$$
a_{11}-\lambda_{q}<r<\lambda_{q}-a_{22} .
$$

Now, let us consider the following $3 \times 3$ non cooperative system

$$
\left\{\begin{array}{l}
L_{q} y_{1}=a_{11} g(x) y_{1}+a_{12} g(x) y_{2}+a_{13} g(x) y_{3}+f_{1} \text { in } \Omega \\
L_{q} y_{2}=a_{21} g(x) y_{1}+a_{22} g(x) y_{2}+a_{23} g(x) y_{3}+f_{2} \text { in } \Omega \\
L_{q} y_{3}=a_{31} g(x) y_{1}+a_{32} g(x) y_{2}+a_{33} g(x) y_{3}+f_{3} \text { in } \Omega \\
y_{1}, y_{2}, y_{3} \rightarrow 0 \text { as } \quad|x| \rightarrow \infty \\
y_{1}=y_{2}=y_{3}=0 \text { on } \partial \Omega
\end{array}\right.
$$

Assume that

$$
a_{12}, a_{13}<0, \quad a_{21}, a_{23}, a_{31}, a_{32} \geq 0
$$

We insert our $3 \times 3$ non-cooperative system into a $4 \times 4$ cooperative one. We introduce the following fourth equation of a new variable $y_{4}=y_{1}-\xi_{2} y_{2}-\xi_{3} y_{3}$ :

$$
\begin{aligned}
L_{q} y_{4}=\left(a_{11}-\xi_{2} a_{21}-\right. & \left.\xi_{3} a_{31}-s\right) g(x) y_{1} \\
+ & \left(a_{12}-\xi_{2} a_{22}-\xi_{3} a_{32}+s \xi_{2}\right) g(x) y_{2} \\
& +\left(a_{13}-\xi_{2} a_{23}-\xi_{3} a_{33}+s \xi_{3}\right) g(x) y_{3}+s g(x) y_{4}+f_{4},
\end{aligned}
$$

where $\xi_{2}$ and $\xi_{3}$ are positive numbers and $f_{4}=f_{1}-\xi_{2} f_{2}-\xi_{3} f_{3}$. Then system $\left(S_{3}\right)$ can be changed into system of the form

$$
\left\{\begin{array}{l}
L_{q} Y=\mathcal{N} g(x) Y+F \quad \text { in } \Omega \\
Y \rightarrow 0 \text { as } \quad|x| \rightarrow \infty \\
Y=0 \text { on } \partial \Omega
\end{array}\right.
$$

where

$$
L_{q}=\left(\begin{array}{cccc}
-\Delta+q & 0 & 0 & 0 \\
0 & -\Delta+q & 0 & 0 \\
0 & 0 & -\Delta+q & 0 \\
0 & 0 & 0 & -\Delta+q
\end{array}\right) \text {, }
$$


$Y=\left(y_{1}, y_{2}, y_{2}, y_{4}\right), F=\left(f_{1}, f_{2}, f_{3}, f_{4}\right)$, and

$$
\mathcal{N}=\left(\begin{array}{cccc}
a_{11}-r & a_{12}+r \xi_{2} & a_{13}+r \xi_{3} & r \\
a_{21} & a_{22} & a_{23} & 0 \\
a_{31} & a_{32} & a_{33} & 0 \\
b_{1} & b_{2} & b_{3} & s
\end{array}\right)
$$

with

$$
\begin{gathered}
b_{1}=a_{11}-\xi_{2} a_{21}-\xi_{3} a_{31}-s, \quad b_{2}=a_{12}-\xi_{2} a_{22}-\xi_{3} a_{32}+s \xi_{2}, \\
b_{3}=a_{13}-\xi_{2} a_{23}-\xi_{3} a_{33}+s \xi_{3} .
\end{gathered}
$$

For the cooperativeness of system $\left(S_{3}^{\prime}\right)$, we can choose $r, s, \xi_{2}$, and $\xi_{3}$ such that

$$
\begin{gathered}
r>0, \quad a_{12}+r \xi_{2}=0, \quad a_{13}+r \xi_{3}=0, \\
a_{11}-\xi_{2} a_{21}-\xi_{3} a_{31}-s=0, \\
a_{12}-\xi_{2} a_{22}-\xi_{3} a_{32}+s \xi_{2}=0, \\
a_{13}-\xi_{2} a_{23}-\xi_{3} a_{33}+s \xi_{3}=0,
\end{gathered}
$$

and then, using (15), we get $\xi_{3}=-\frac{a_{13}}{r}>0$ and $\xi_{2}=-\frac{a_{12}}{r}>0$. Now, system $\left(S_{3}^{\prime}\right)$ satisfies the maximum principle if the matrix

$$
\left(\begin{array}{cccc}
\lambda_{q}-a_{11}+r & -a_{12}-r \xi_{2} & -a_{13}-r \xi_{3} & -r \\
-a_{21} & \lambda_{q}-a_{22} & -a_{23} & 0 \\
-a_{31} & -a_{32} & \lambda_{q}-a_{33} & 0 \\
-b_{1} & -b_{2} & -b_{3} & \lambda_{q}-s
\end{array}\right)
$$

is a non-singular $M$-matrix, which means that

$$
\begin{gathered}
\lambda_{q}-a_{11}+r>0 \\
\left|\begin{array}{cc}
\lambda_{q}-a_{11}+r & -a_{12}-r \xi_{2} \\
-a_{21} & \lambda_{q}-a_{22}
\end{array}\right|>0, \\
\left|\begin{array}{ccc}
\lambda_{q}-a_{11}+r & a_{12}+r \xi_{2} & -a_{13}-r \xi_{3} \\
-a_{21} & \lambda_{q}-a_{22} & -a_{23} \\
-a_{31} & -a_{32} & \lambda_{q}-a_{33}
\end{array}\right|>0,
\end{gathered}
$$

and

$$
\left|\begin{array}{cccc}
\lambda_{q}-a_{11}-r & -a_{12}-r \xi_{2} & -a_{13}-r \xi_{3} & -r \\
-a_{21} & \lambda_{q}-a_{22} & -a_{23} & 0 \\
-a_{31} & -a_{32} & \lambda_{q}-a_{33} & 0 \\
-b_{1} & -b_{2} & -b_{3} & \lambda_{q}-s
\end{array}\right|>0 .
$$

Therefore, using (17)-(20), we obtain

$$
\begin{gathered}
\lambda_{q}-a_{11}+r>0, \quad\left(\lambda_{q}-a_{11}+r\right)\left(\lambda_{q}-a_{22}\right)>0, \\
\left(\lambda_{q}-a_{11}+r\right)\left[\left(\lambda_{q}-a_{22}\right)\left(\lambda_{q}-a_{33}\right)-a_{23} a_{32}\right]>0,
\end{gathered}
$$


and

$$
\left(\lambda_{q}-a_{11}+r\right)\left(\left(\lambda_{q}-a_{22}\right)\left(\lambda_{q}-a_{33}\right)\left(\lambda_{q}-s\right)-a_{23} a_{32}\left(\lambda_{q}-s\right)\right)>0,
$$

which implies that

$$
\lambda_{q}-a_{11}+r>0, \quad \lambda_{q}-a_{22}>0, \quad\left(\lambda_{q}-a_{22}\right)\left(\lambda_{q}-a_{33}\right)>a_{23} a_{32}, \quad \lambda_{q}-s>0 .
$$

From (15), we obtain $\left(\lambda_{q}-a_{22}\right)\left(\lambda_{q}-a_{33}\right)>0$ and, thus,

$$
a_{11}-r<\lambda_{q}, \quad a_{22}<\lambda_{q}, \quad a_{33}<\lambda_{q}, \quad s<\lambda_{q} .
$$

It follows from (17) and (19) that

$$
a_{22}+r+\frac{\xi_{3}}{\xi_{2}} a_{32}=s,
$$

whereas (17) and (20) yield that

$$
a_{33}+r+\frac{\xi_{2}}{\xi_{3}} a_{23}=s
$$

Since $s<\lambda_{q}$, from (21) and (22) we obtain

$$
\lambda_{q}>a_{22}+r+\frac{\xi_{3}}{\xi_{2}} a_{32}, \quad \lambda_{q}>a_{33}+r+\frac{\xi_{2}}{\xi_{3}} a_{23},
$$

which, in view of (15), guarantee that

$$
\lambda_{q}>a_{22}+r \quad \lambda_{q}>a_{33}+r,
$$

i. e.,

$$
r<\lambda_{q}-a_{22}, \quad r<\lambda_{q}-a_{33}, \quad \text { and } \quad r>a_{11}-\lambda_{q} .
$$

Consequently, we have

Theorem 5. Assume that (15) holds. Then for $0 \leq \xi_{3} f_{3} \leq \xi_{2} f_{2} \leq f_{1}$, system $\left(S_{3}\right)$ satisfies the generalized maximum principle if

$$
a_{11}-\lambda_{q}<r<\lambda_{q}-a_{22}, \quad a_{11}-\lambda_{q}<r<\lambda_{q}-a_{33} .
$$

For non-cooperative system (S), we set the following conditions:

$$
\begin{array}{ll}
a_{1 j}<0 & \text { for } \quad j=2,3, \ldots, n, \\
a_{i j} \geq 0 & \text { for } \quad i=2,3, \ldots, n, j=1,2, \ldots, n, i \neq j .
\end{array}
$$

Similarly, to construct $(n+1) \times(n+1)$ cooperative system, we introduce the following equation of a new variable $y_{n+1}=y_{1}-\sum_{i=2}^{n} \xi_{i} y_{i}$, where $\xi_{2}, \ldots, \xi_{n}$ are positive numbers and thus we have

Theorem 6. Assume that (23) holds. Then for $0 \leq \xi_{n} f_{n} \leq \cdots \leq \xi_{2} f_{2} \leq f_{1}$, system (S) satisfies the generalized maximum principle if

$$
a_{11}-\lambda_{q}<r<\lambda_{q}-a_{i i} \text { for all } i=2,3, \ldots, n .
$$




\section{ACKNOWLEDGEMENT}

The authors would like to express their gratitude to Professor Jacqueline Fleckinger (QREMAQ - Université Toulouse 1, France) for her constructive suggestions.

\section{REFERENCES}

[1] A. Abakhti-Mchachti and J. Fleckinger-Pellé, "Existence of positive solutions for noncooperative semilinear elliptic system defined on an unbounded domain," in Progress in partial differential equations: elliptic and parabolic problems (Pont-à-Mousson, 1991), ser. Pitman Res. Notes Math. Ser. Harlow: Longman Sci. Tech., 1992, vol. 266, pp. 92-106.

[2] B. Alziary, N. Besbas, L. Cardoulis, and J. Fleckinger, "Maximum and antimaximum principles for some elliptic systems involving Schrödinger operators," in Partial differential equations, ser. Lecture Notes in Pure and Appl. Math. New York: Dekker, 2002, vol. 229, pp. 13-30.

[3] B. Alziary, L. Cardoulis, and J. Fleckinger-Pellé, "Maximum principle and existence of solutions for elliptic systems involving Schrödinger operators," Rev. R. Acad. Cienc. Exactas Fís. Nat. (Esp.), vol. 91, no. 1, pp. 47-52, 1997.

[4] B. Alziary, J. Fleckinger-Pellé, and P. Takáč, "An extension of maximum and anti-maximum principles to a Schrödinger equation in $\mathbb{R}^{2}$, , J. Differential Equations, vol. 156, no. 1, pp. 122-152, 1999. [Online]. Available: http://dx.doi.org/10.1006/jdeq.1998.3609

[5] M. Bouchekif, H. Serag, and F. de Thélin, "On maximum principle and existence of solutions for some nonlinear elliptic systems," Rev. Mat. Apl., vol. 16, no. 1, pp. 1-16, 1995.

[6] P. Clément, D. G. de Figueiredo, and E. Mitidieri, "Positive solutions of semilinear elliptic systems," Comm. Partial Differential Equations, vol. 17, no. 5-6, pp. 923-940, 1992. [Online]. Available: http://dx.doi.org/10.1080/03605309208820869

[7] D. G. de Figueiredo and E. Mitidieri, "Maximum principle for linear elliptic systems," Quaterno Matematico, 177, Dip. Sc. Mat., Univ. Trieste, 1988.

[8] D. G. de Figueiredo and E. Mitidieri, "A maximum principle for an elliptic system and applications to semilinear problems," SIAM J. Math. Anal., vol. 17, no. 4, pp. 836-849, 1986. [Online]. Available: http://dx.doi.org/10.1137/0517060

[9] D. G. de Figueiredo and E. Mitidieri, "Maximum principles for cooperative elliptic systems," C. R. Acad. Sci. Paris Sér. I Math., vol. 310, no. 2, pp. 49-52, 1990.

[10] A. Djellit and A. Yechoui, "Existence of principal eigenvalues for some boundary value problems," Seminaire d'analyse E.D.P., CEREMATH, Univ. Toulouse 1, France, 1996-1997.

[11] J. Fleckinger, J. Hernández, and F. de Thélin, "Principe du maximum pour un système elliptique non linéaire,” C. R. Acad. Sci. Paris Sér. I Math., vol. 314, no. 9, pp. 665-668, 1992.

[12] J. Fleckinger-Pellè and H. Serag, "On maximum principle and existence of solutions for elliptic systems on $\mathbb{R}^{n}$," J. Egyptian Math. Soc., vol. 2, pp. 45-51, 1994.

[13] J. Fleckinger-Pellé and H. Serag, "Semilinear cooperative elliptic systems on $\mathbb{R}^{n}$," Rend. Mat. Appl. (7), vol. 15, no. 1, pp. 89-108, 1995.

[14] J. Fleckinger-Pellé and P. Takáč, "Uniqueness of positive solutions for nonlinear cooperative systems with the p-Laplacian,” Indiana Univ. Math. J., vol. 43, no. 4, pp. 1227-1253, 1994. [Online]. Available: http://dx.doi.org/10.1512/iumj.1994.43.43053

[15] I. M. Gali and H. M. Serag, "Optimal control of cooperative elliptic systems defined on $\mathbb{R}^{n}$," J. Egyptian Math. Soc., vol. 3, pp. 33-39, 1995.

[16] H. M. Hassan and H. M. Serag, "Optimal control for quasi-static problem with viscous boundary conditions," Indian J. Pure Appl. Math., vol. 31, no. 7, pp. 767-772, 2000. 
[17] J.-L. Lions, Optimal control of systems governed by partial differential equations., ser. Translated from the French by S. K. Mitter. Die Grundlehren der mathematischen Wissenschaften, Band 170. New York: Springer-Verlag, 1971.

[18] H. M. Serag, "On optimal control for elliptic system with variable coefficients," Rev. Mat. Apl., vol. 19, no. 1-2, pp. 37-41, 1998.

[19] H. M. Serag, "Distributed control for cooperative systems governed by Schrodinger operator," J. Discrete Math. Sci. Cryptography, vol. 3, no. 1-3, pp. 227-234, 2000.

[20] H. M. Serag, "Optimal control of systems involving Schrödinger operators," Control Intell. Syst., vol. 32, no. 3, pp. 154-157, 2004.

[21] H. M. Serag and A. H. Qamlo, "On elliptic systems involving Schrödinger operators," Mediterranean Journal of Measurement and Control, vol. 1, no. 2, pp. 91-96, 2005.

[22] H. M. Serag and E. A. El-Zahrani, "Maximum principle and existence of positive solutions for nonlinear systems on $\mathbb{R}^{N}$," Electron. J. Differential Equations, pp. No. 85, 12 pp. (electronic), 2005.

\section{Authors' addresses}

\section{H. M. Serag}

Mathematics Department, Faculty of Science, Al-Azhar University, 11884 Nasr City, Cairo, Egypt

E-mail address: serraghm@yahoo.com

\section{A. H. Qamlo}

Mathematics Department, Faculty of Education for Girls, Makka, P.O. Box 3653, Saudi Arabia 